\title{
Complete Deletion of the Neurotrophin Receptor p75 NTR Leads to Long-Lasting Increases in the Number of Basal Forebrain Cholinergic Neurons
}

\author{
Thomas Naumann, ${ }^{1 *}$ Elisabeth Casademunt, ${ }^{2 *}$ Ewald Hollerbach, ${ }^{1}$ Jutta Hofmann, ${ }^{1}$ Georg Dechant, ${ }^{2}$ \\ Michael Frotscher, ${ }^{1}$ and Yves-Alain Barde ${ }^{2}$ \\ 1/nstitute of Anatomy, University of Freiburg, D-79104 Freiburg, Germany, and 2Department of Neurobiology, Max Planck \\ Institute of Neurobiology, D-82152 Martinsried, Germany
}

Cholinergic neurons innervating cortical structures are among the most affected neuronal populations in Alzheimer's disease. In rodents, they express high levels of the neurotrophin receptor $\mathrm{p} 75^{\mathrm{NTR}}$. We have analyzed cholinergic septohippocampal neurons of the medial septal nucleus in p75 exonIII (partial p75 ${ }^{\text {NTR }}$ knock-out) and $p 75^{\text {exonIV }}$ (complete p75 NTR knock-out) mice, in their original genetic background and in congenic strains. At postnatal day 15 , the $p 75^{\text {exonlll }}$ mutation leads to a moderate increase $(+13 \%)$ in these neurons among littermates only after back-crossing in a C57BL/6 background. In contrast, the null $p 75^{\text {exonIV }}$ mutation, which prevents expression of both the full- length and the shorter p $75^{\mathrm{NTR}}$ isoforms, results in a $28 \%$ neuronal increase, independent of genetic background. The incomplete nature of the $\mathrm{p} 75^{\mathrm{NTR}}$ mutation used previously, coupled with difficulties in delineating the mouse medial septum and the impact of the genetic background on cell numbers, all contribute to explain previous difficulties in establishing the role of $p 75^{N T R}$ in regulating cholinergic neuron numbers in the mouse forebrain.

Key words: p75 NTR; cholinergic neurons; medial septum; cell death; genetic background; optical fractionator; NGF
Large neurons of the basal forebrain give rise to the widespread cholinergic innervation of cortical structures. The most widely investigated population of these neurons, located in the septum and projecting to the hippocampal formation (Sofroniew et al., 1987), is used frequently as a model for CNS cholinergic neurons. In Alzheimer's disease, the loss of explicit memory is accompanied by a loss of cholinergic function (Bartus et al., 1982; Coyle et al., 1983; Perry et al., 1999). In addition, learning-deficient rats have smaller basal forebrain cholinergic neurons, and their performance, as well as the size of their cholinergic neurons, can be increased by the administration of nerve growth factor (Fischer et al., 1987). In view of the functional importance of cholinergic neurons, it is of considerable interest to understand the molecular mechanisms regulating their survival.

The neurotrophins are among the best studied signaling systems regulating neuronal numbers in the nervous system (Bibel and Barde, 2000). They are well known to affect the viability, differentiation, and size of CNS cholinergic neurons (Hefti, 1986; Vantini et al., 1989; Li et al., 1995; Lucidi-Phillipi et al., 1996). NGF and brain-derived neurotrophic factor are synthesized by hippocampal neurons, which are the target cells of forebrain cholinergic neurons. NGF is taken up by the cholinergic terminals of the septohippocampal neurons bearing both $\mathrm{p} 75^{\mathrm{NTR}}$ and TrkA

Received Sept. 24, 2001; revised Nov. 30, 2001; accepted Dec. 10, 2001.

This study was supported by the Deutsche Forschungsgemeinschaft (A1, SFB 505) and the European Union (projects PL960024 and QLRT-1999-00602 to Y.B.). We thank Dr. J. Schulte-Mönting (Institute for Medical Informatics, University of Freiburg) for statistical analysis and O. Segun for management of our mouse colony.

*T.N. and E.C. contributed equally to this study.

Correspondence should be addressed to Dr. Thomas Naumann, Institute of Anatomy I, Albertstraße 17, D-79104 Freiburg, Germany. E-mail: naumannt@ uni-freiburg.de.

Y.-A. Barde's present address: Friedrich Miescher Institute for Biomedical Research, Maulbeerstraße 66, CH-4058 Basel, Switzerland.

Copyright $\odot 2002$ Society for Neuroscience $0270-6474 / 02 / 222409-10 \$ 15.00 / 0$ receptors and is retrogradely transported to the cell body region (for review, see Hagg et al., 1994). Although the septal neurons have long been known to express $\mathrm{p} 75^{\mathrm{NTR}}$ at particularly high levels, the role of this receptor in their survival is unclear and controversial. Van der Zee et al. (1996) first reported an increased number of cholinergic neurons in the septum of mice carrying a targeted mutation in the third exon of the $p 75^{N T R}$ gene. However, subsequent investigations using the same mouse mutant led to conflicting results, with some studies indicating an increase and others indicating a decrease or no significant changes (Hagg et al., 1997; Peterson et al., 1997; Yeo et al., 1997; Hagg, 1999; Peterson et al., 1999; Ward and Hagg, 1999; Greferath et al., 2000).

Recently, a new mutation in the $p 75^{N T R}$ locus was generated that also eliminates a splice variant encoded by this locus (von Schack et al., 2001). This previously unrecognized variant encodes a "short" form of p75 NTR (s-p75 NTR) that has the same transmembrane and cytoplasmic domain as the full-length, well characterized form of $\mathrm{p} 75^{\mathrm{NTR}}$. Mice bearing this novel $p 75^{\text {exonIV }}$ mutation display a more drastic phenotype than the previous p $75^{\text {exonIII }}$ mutants (Lee et al., 1992), including a larger reduction in the number of dorsal root ganglia neurons and Schwann cells. This new $p 75^{N T R}$ mutant was used in the present study, and because we wished to compare the results with those obtained using the previous, hypomorphic $p 75^{N T R}$ mutation, we also generated congenic strains carrying each of the two mutations. For each mouse strain, we also defined the boundaries of the medial septum (MS) by retrograde Fluoro-Gold (FG) tracing in combination with immunolabeling for ChAT and $\mathrm{p} 75^{\mathrm{NTR}}$.

\section{MATERIALS AND METHODS}

Mouse strains and breeding colony. The $p 75^{\text {exonIII }}$ mutation was generated as described by Lee et al. (1992) using J1(129) embryonic stem (ES) cells 


\section{Strains used : generation and genetic background}

C57BL/6NJCrI (B6) from Charles River Labs; inbred at the MPI for multiple generations

p75 $^{\text {exonllI }}$ : (1) strain JR2124 from Jackson Labs, inbred at the MPI for several generations

(2) backcrossed into $\mathrm{B} 6>7$ generations; >95\% B6

p75 $^{\text {exon/v }}$ : (3) mixed background; $50 \%$ B6/Sv129 mixture

(4) Backcrossed into B6 >7 generations; > 95\% B6

B

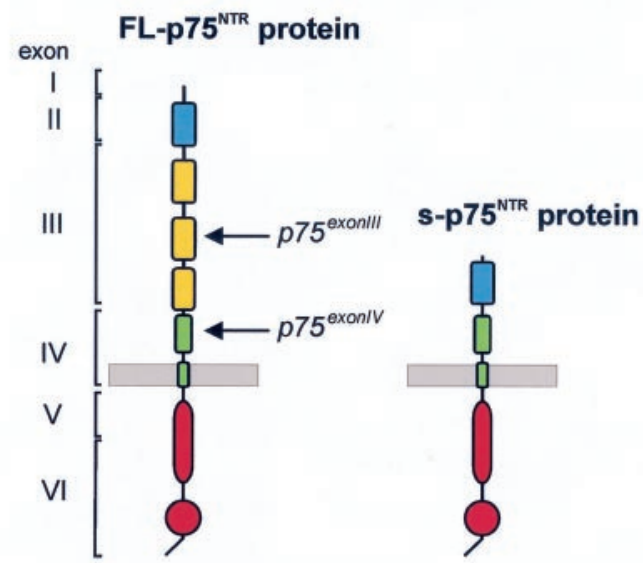

C



Figure 1. Mutations and mouse strains used in this study. $A$, List of the five strains analyzed and their origins. $B$, Schematic representation of the full-length $\mathrm{p} 75^{\mathrm{NTR}}$ and the splice variant s-p $75^{\mathrm{NTR}}$ proteins, depicting their different domains and their correlation to the intron-exon structure of the genomic locus. Arrows point to the approximate location of the targeting event: the $p 75^{\text {exonIII }}$ mutation (Lee et al., 1992) replaced part of exon III with a selection cassette, whereas the $p 75^{\text {exonIV }}$ mutation inserted the cassette in reverse orientation within exon IV, thus preventing expression of both the fulllength and the s-p75 NTR splice isoform (von Schack et al., 2001). $C$, Breeding scheme for the $p 75^{\text {exonIII }}$ line (left) and generation of a congenic B6 strain bearing this mutation. The original line obtained from The Jackson Laboratory was maintained by heterozygous matings and crosses to Sv129, resulting in a line of mixed background in which Sv129 is most prominent. To generate the congenic B6 strain (right), originally mixed-background heterozygotes were consecutively mated to B6 males from Charles River Laboratories. The congenic $p 75^{\text {exonIV }}(\mathrm{B} 6)$ strain was generated according to the same scheme.

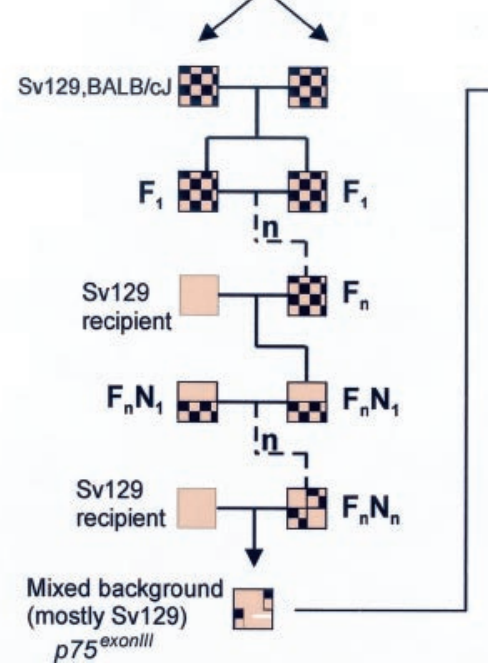

Generation of congenic B6 strains

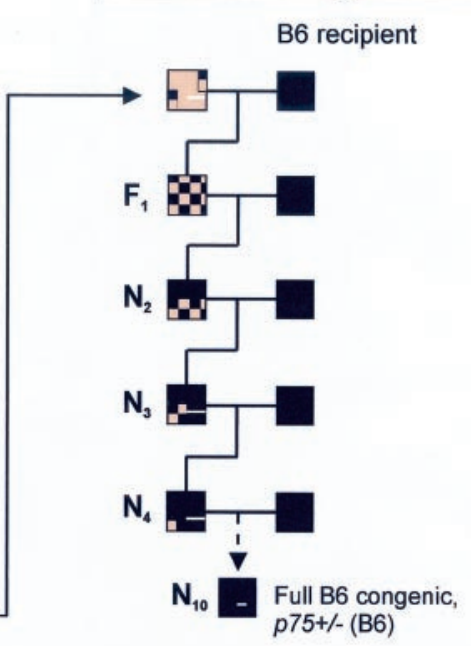

and was obtained from The Jackson Laboratory (Bar Harbor, ME) (strain JR2124) in its original, mixed background, Sv129/BALB/cJ. It was maintained at the Max Planck Institute by alternating breeding to Sv129 (Sv129Pas substrain) to generate heterozygotes and breeding of heterozygous siblings, as shown schematically in Figure $1 C$.

The $p 75^{\text {exonIV }}$ mutation was generated as described previously (von Schack et al., 2001) in R1(129) ES cells/C57BL/6 blastocysts and kept in a mixed genetic background by breeding of heterozygous siblings. This line was at F5 (fifth generation) at the time of submission of this manuscript.

Congenic C57BL/6 (B6) strains bearing each of the two mutations were generated by consecutive mating of a heterozygous mouse in the mixed background to a pure B6 mouse obtained from Charles River Laboratories (Wilmington, MA). All mice analyzed for this report were from at least the seventh backcross generation.
Genetic (microsatellite marker) analysis. Tail genomic DNA was extracted from the following three representative mice: a B6 mouse from Charles River Laboratories, a $p 75^{\text {exonIII }}(\mathrm{B} 6)$ mouse, and a $p 75^{\text {exonIV }}(\mathrm{B} 6)$ mouse of the same backcross generation. Fifty-nine PCRs spanning microsatellite regions in the entire mouse genome were performed on each genomic DNA. PCR primer sequences were taken from the Mouse Genome Database (www.informatics.jax.org); licensed oligonucleotides were from Research Genetics (Huntsville, AL). In each lane, ROX 500 (Applied Biosystems, Foster City, CA) was used as a size standard. The fragment length of each amplicon was analyzed using an Applied Biosystems Prism 377 DNA Sequencer; gel analysis and determination of the fragment length were done using the GeneScan and GenoTyper software packages (Applied Biosystems). Fragment lengths were then compared with data available for C57BL/6 and Sv129 mice from Jackson and Charles River Laboratories (Medigenomix, Martinsried, Germany). 


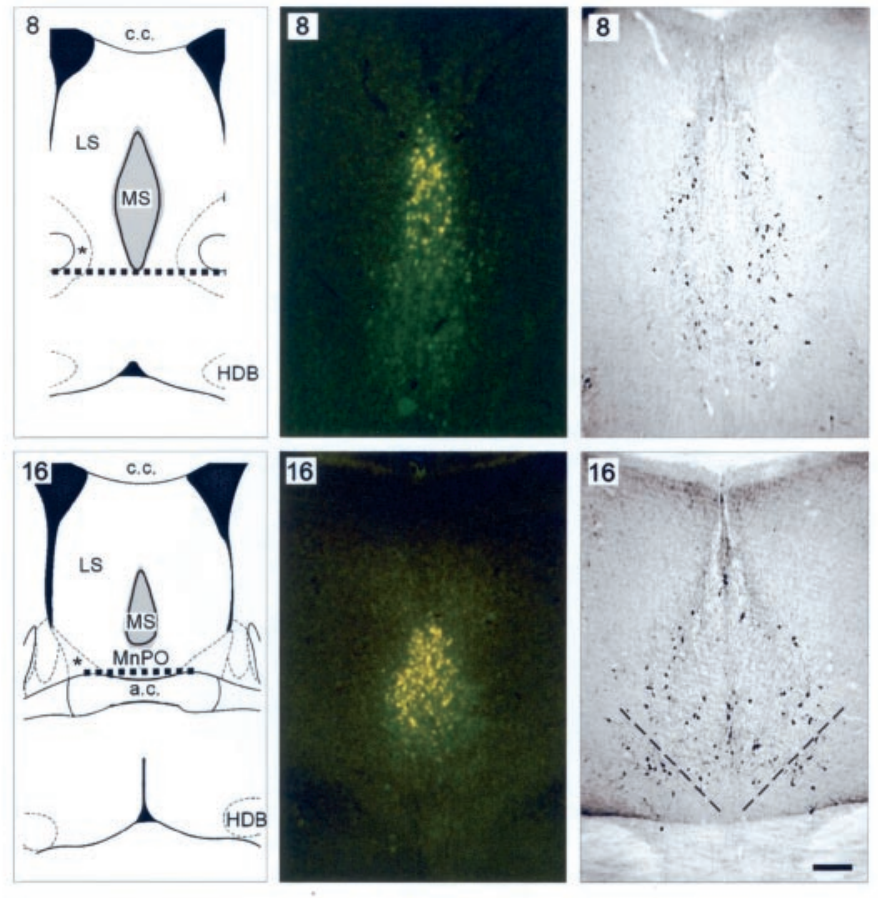

Abbreviations used in figures:

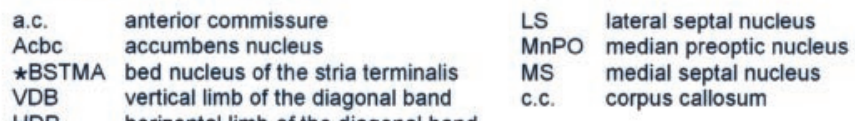

Figure 2. Retrograde FG tracing for septohippocampal neurons to delineate the actual boundaries of the MS. Five coronal sections of the septal complex from a $p 75^{\text {exonIV }}$ (Sv129/B6) mutant after intrahippocampal injections of the retrograde fluorescent tracer FG (middle columns) and subsequent immunocytochemical detection of ChAT-positive cholinergic neurons in the same sections (right columns) are shown. The actual position is indicated using section numbers according to our nomenclature described in Figure 3. Left, A schematic anatomic diagram (adapted from Franklin and Paxinos, 1997) of the estimated position. The shaded area represents the MS region in which FG-backlabeled cholinergic neurons projecting to the hippocampal formation were identified. The dotted line connecting the inferior edge of the anterior commissure marks the inferior boundary of the area in which neurons were counted. Note that the extent of the shaded area (and therefore the location of cholinergic MS neurons to be counted) changes substantially along the rostrocaudal axis of the MS. Oblique, dashed lines in the last panel (position 16) indicate the boundary between MS and neighboring ventrocaudal nuclei not analyzed in this study.

$F G$ retrograde tracing. Because neighboring septal nuclei (e.g., the lateral septal nucleus) also contain cholinergic neurons, which only exceptionally colocalize with $\mathrm{p} 75^{\mathrm{NTR}}$, tracing experiments were performed to delineate the boundaries of the region containing cholinergic septohippocampal projection neurons of each strain. Wild-type and mutant mice received intrahippocampal injections of the retrograde fluorescent tracer FG bilaterally (four injections each, 2.5\% FG) (Naumann et al., 1992). One week later, mice were perfused and brains were cut as described below. Vibratome sections containing the septal region were first analyzed using fluorescence microscopy to define the "region of interest" (Fig. 2). Sections containing FG-backlabeled septohippocampal neurons were first photodocumented and then immunostained against ChAT (Fig. 2) or p75 NTR.

Tissue processing and immunocytochemistry. Mice were transcardially perfused first with $0.9 \%$ saline and then with $4 \%$ paraformaldehyde in $0.1 \mathrm{M}$ phosphate buffer ( $\mathrm{PB}, \mathrm{pH} 7.35)$. Whole brains were post-fixed in the same fixative for $2 \mathrm{hr}$. Coronal $50 \mu \mathrm{m}$ sections were cut on a vibratome across the entire septal region and collected to reconstruct the complete series as described in detail in Results (see also Fig. 3).

ChAT immunocytochemistry was performed with a goat anti-ChAT polyclonal antibody (1:500 in $0.1 \mathrm{M}$ PB containing 5\% normal rabbit serum and $0.5 \%$ Triton X-100; Bioproducts, Boehringer Ingelheim, Ingelheim, Germany) for $48 \mathrm{hr}$ at $4^{\circ} \mathrm{C}$, followed by biotinylated rabbit anti-goat IgG. p75 NTR immunocytochemistry (data not shown) was performed using an anti-human antibody (catalog \#G3231; Promega, Madison, WI) diluted 1:1000 in $0.1 \mathrm{M} \mathrm{PB}$ containing $1 \%$ normal goat serum and $0.5 \%$ Triton $\mathrm{X}-100$. Immunostaining was visualized as described previously (Naumann et al., 1997) using the avidin-biotin complex Elite Kit (Vector Laboratories, Burlingame, CA) followed by DAB reaction. Every second section of each complete series (Fig. 3) was used for statistical analysis. Sections were mounted on slides, dehydrated, and coverslipped using Histokit (Shandon, Pittsburgh, PA).

Cell counts: quantitative stereology and statistical analysis. The number of cholinergic MS neurons was first obtained by direct, "manual" counts on ChAT-immunostained sections (Naumann et al., 1994) and subsequently with the optical disector/fractionator method (OF; see also West et al., 1991), but relative differences among groups were directly comparable (within 3\%) using both methods. Figures 4-6 show only stereological data obtained by one observer with the OF, because this method is unbiased and is currently the method of choice (Peterson et al., 1999). 
Figure 3. Variability in the localization of the MS nucleus in $p 75^{\text {exonIII }}$ and p $75^{\text {exonIV }}$ mutants. Representative examples of the morphological analysis performed for each mutation in the different backgrounds and at two different ages are shown. In each panel, the mutant mice are represented in the top half, and the corresponding wild-type littermates are represented in the bottom half. In each individual panel, mice belonging to the same litter are represented by the same color. All frontal sections of the entire septal complex were collected as complete series and numbered according to their exact position from rostral (left) to caudal (right) positions (vertical lines in each panel). Independent of malformations of the fiber tracts, the coronal section through the septal complex was designated section c.c., where the tips of the corpus callosum were first found in close contact (Fig. 2). Because every second section of the complete series was used for quantitative stereological analysis, each position refers to the "0" position of the c.c., using even numbers in both directions. Each horizontal bar represents the complete series of the septal complex sections containing cholinergic MS neurons in an individual mouse. Numbers on each bar (e.g., 4959/22) indicate the total number of ChAT-immunoreactive neurons counted in the MS (4959) and the number of sections collected through the MS (22). In some cases, virtually no cholinergic neuron was detectable in the most caudal section of the MS region (hatched region of the horizontal bar). Note the higher degree of variability in the $p 75^{\text {exonIII }}$ line than the $p 75^{\text {exonIV }}$ line, both at $\mathrm{P} 15$ and in the adult.

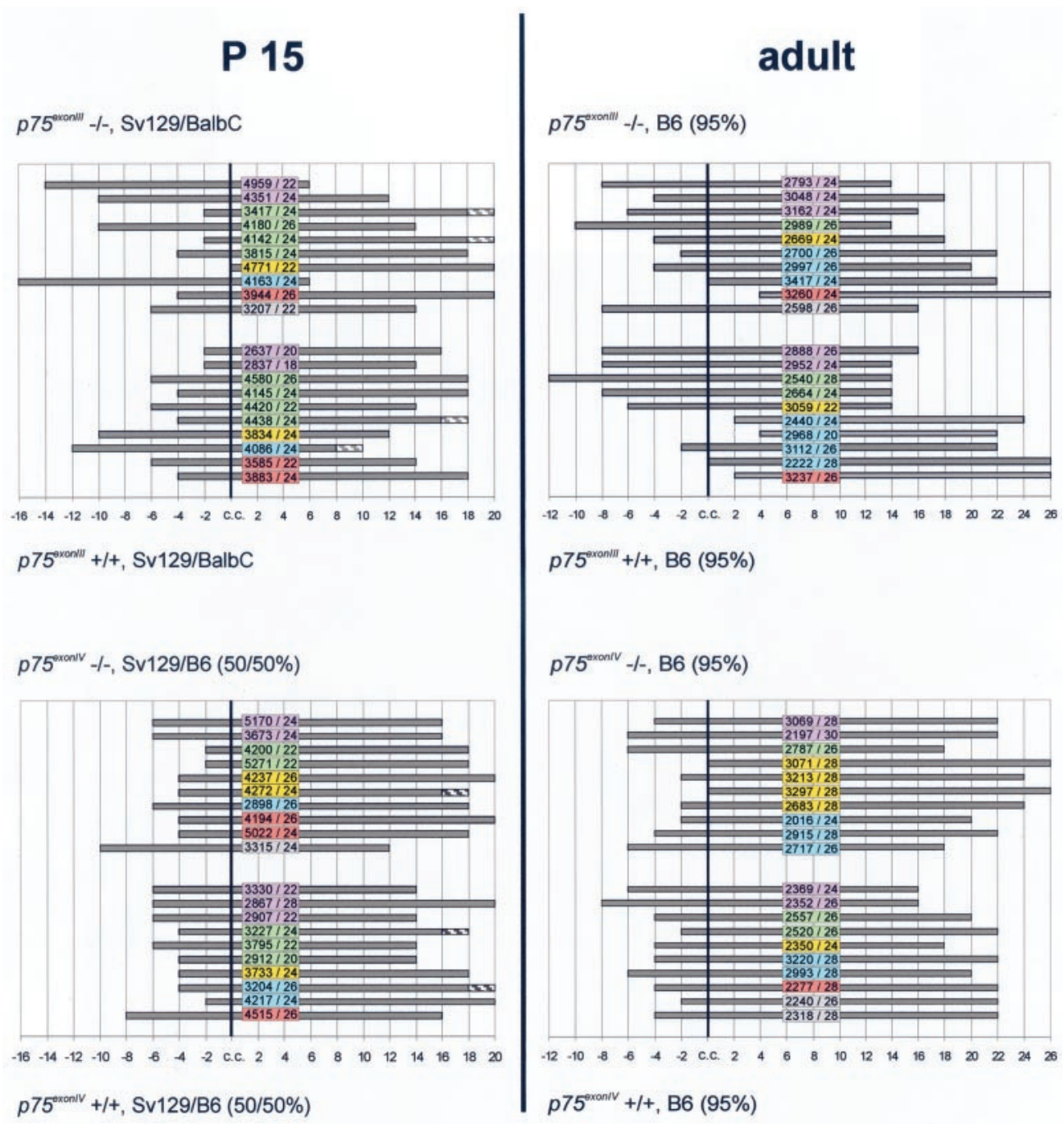

For quantitative stereology, sections of the septal region were visualized on a computer screen attached to an Olympus BX60 microscope F5 (Olympus Optical Co. Ltd., Düsseldorf, Germany). A computercontrolled stepper motor stage and focus assembly allowed movement in the $x$-, $y$-, and $z$-axes. Cell counts were performed using Stereo Investigator software (version 3.0; MicroBrightField, Inc., Colchester, VT). According to our criteria described in Figures 2 and 3, the region of interest was first marked for every single section using low-power magnification $(4 \times / 0.10$ objective $)$. For subsequent cell counts, the following parameters were added to the program: counting frame, $50 \times 30 \mu \mathrm{m}$; guard zone, $2 \mu \mathrm{m}$; and counting depth, $8 \mu \mathrm{m}$. Thereafter, using highpower magnification (oil objective lens, $100 \times / 1.35$ ), ChAT-positive cells that fulfilled the criteria of the unbiased counting rules were marked and added to the probe run list. The total cell numbers estimated by the OF were subsequently analyzed by three-way ANOVA (6.12 PROC GLM; SAS Institute, Cary, NC). Statistical significance was analyzed for the corresponding three classes with two levels each [1, wild type/knock-out; 2 , $p 75^{\text {exonIII }} / p 75^{\text {exonIV }}$ mutation; 3 , original background/B6 or postnatal day 15 (P15)/adult], all with repeated measurements.

Reverse transcription-PCR analysis. cDNA was synthesized from $1 \mu \mathrm{g}$ of total RNA from whole brain and from the MS region of P15 mice (B6 and Sv129 strains) as described previously (von Schack et al., 2001). The following primers were used to detect FL-p75 ${ }^{\mathrm{NTR}}$ : $5^{\prime}$-CCT GCC TGG ACA GTG TTA CG-3' and 5'-GCC AAG ATG GAG CAA TAG ACA G-3'; 5'-TGC CTG GAC AAG ATC CCT GG-3' and 5'-GGC CTG AGG CAG TCT GTG TG-3' were used to detect s-p $75^{\text {NTR }}$. Detection of the s-p $75^{\mathrm{NTR}}$ transcript requires at least 2.5 -fold higher levels of input cDNA than detection of FL-p $75^{\text {NTR }}$.

\section{RESULTS}

\section{Genetic manipulations: $p 75^{N T R}$ mutations and generation of congenic strains}

The $p 75^{\text {exonIII }}$ mutation was first reported in 1992 (Lee et al., 1992) and was initially distributed by The Jackson Laboratory (strain JR2124) in a mixed Sv129/BALB/cJ background after consecutive matings of homozygous siblings. This mutation targeted exon III (Fig. 1) and does not affect the expression of a splice variant of $\mathrm{p} 75^{\mathrm{NTR}}$ (s-p $75^{\mathrm{NTR}}$ ) that has only been discovered recently (von Schack et al., 2001). The $p 75^{\text {exonIV }}$ mutation, which eliminates expression of both the full-length $\mathrm{p} 75^{\mathrm{NTR}}$ receptor and the s-p75 ${ }^{\text {NTR }}$ isoform (Fig. $1 B$ ), was generated in a mixed Sv129/C57BL/6 background (von Schack et al., 2001). Originally, then, both lines contained $\sim 50 \%$ of Sv129 background in their genomes. The Sv129 background has been shown to be highly heterogeneous, however, with multiple substrains identified (Simpson et al., 1997). In addition, it is well known that both BALB/c and Sv129 mice display some abnormalities, including malformations of the corpus callosum (c.c.) (Wahlsten, 1982), a crucial landmark for the anatomic definition of the boundaries of the septal nuclei. Finally, the viability, litter size, and reproductive efficacy of the Sv129 strain are known to be rather low (Green and Witham, 1991). We chose the B6 strain to generate congenic 




Figure 4. Variability in the number of ChAT-immunoreactive MS neurons in different wild-type strains. The number of MS cholinergic neurons in wild-type mice from six different lines was quantified using the criteria described in the legends to Figures 2 and 3. Numbers on each bar indicate mean values from 10 animals; error bars indicate SD. In general, a higher B6 content in the genome correlates with a lower number of ChATimmunoreactive MS neurons.

lines (Fig. $1 C$ ) and introduced each of the two p $75^{N T R}$-targeted mutations in the inbred C57BL/6NJCrl background provided by Charles River Laboratories. Although the $p 75^{\text {exonIII }}$ mutation has been inbred at our institute for multiple generations, with occasional crosses to the inbred strain Sv129Pas (Fig. 1C), the mixed B6/Sv129 background of the $p 75^{\text {exonIV }}$ mutation has been better preserved because of the limited (F4, F5) number of generations.

Genomic DNA from a seventh-generation mouse in each line (with an estimated $>98 \%$ B6 content) was subjected to an extensive microsatellite marker analysis and compared with the genomic DNA of a pure B6 mouse. This analysis revealed that the background of the $p 75^{\text {exonIII }}(\mathrm{B} 6)$ mice is almost identical to that of $p 75^{\text {exonIV }}(\mathrm{B} 6)$ mice. As seen in Table 1, both congenic B6 lines display the B6 genotype for the vast majority of the markers tested, with the $p 75^{\text {exonIII }}$ line showing B6/Sv129 heterozygosis at only two positions (D10Mit 205 and D11Mit 270) and the $p 75^{\text {exonIV }}$ line at one unique position (D13Mit 236).

\section{Anatomic analysis: distribution of septohippocampal neurons in the MS}

We analyzed the distribution of septohippocampal projection neurons after intrahippocampal injections of the retrograde flu-

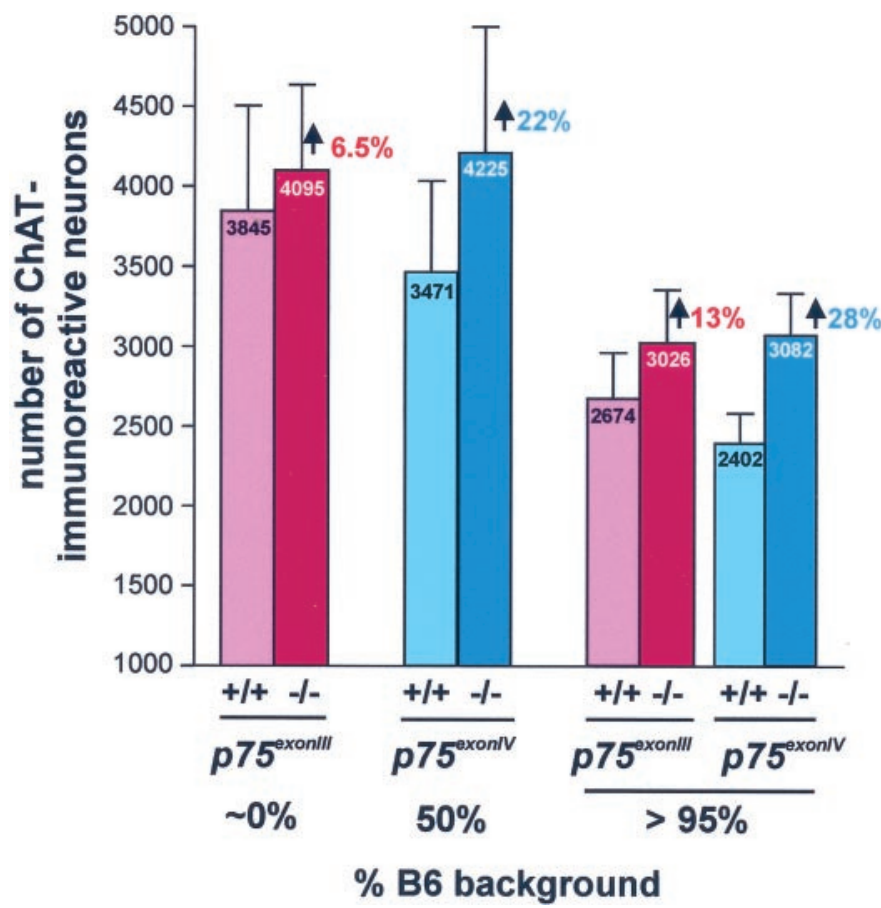

Figure 5. Effect of the two $p 75^{N T R}$ mutations at P15. The effect of each mutation in a mixed background (left) or in congenic B6 strains (right) was quantified as described in Figures 2 and 3. For each line, 10 wild-type and 10 homozygous mutant mice from heterozygous matings were analyzed at P15. The moderate effect of the $p 75^{\text {exonIII }}$ mutation in the mixed background $(6.5 \%$ increase) is enhanced in the B6 background $(13 \%$ increase). In contrast, the $p 75^{\text {exonIV }}$ mutation, which prevents expression of both the full-length receptor and the s-p75 ${ }^{\mathrm{NTR}}$ isoform, leads to comparable effects in both genetic backgrounds (28\% increase in B6 and 22\% increase in a mixed background).

orescent tracer FG in the different mouse strains, including the mutants. As shown in Figure 2 (middle columns), the overall distribution of septohippocampal projection neurons resembles the pattern observed in rats (Naumann et al., 1992), regardless of malformations or partial lack of the corpus callosum. Especially in animals with an Sv129 genetic background, a reduction of fiber crossing between the two hemispheres was often observed. In several cases (primarily in the congenic B6 strains), we also found variable locations of the fimbria-fornix bundle in our serial sections through the caudal region of the medial septum/diagonal band (MSDB) complex. These observations were made both in mutant animals and in their wild-type littermates, suggesting that these malformations are a characteristic feature in a given background (Wahlsten, 1982; Bentivoglio et al., 1994; Wahlsten and Bulman-Fleming, 1994) rather than induced by the mutations in the $p 75^{N T R}$ locus (Peterson et al., 1999). In all animals investigated, we first analyzed the distribution of FG-labeled neurons along the rostrocaudal extent of the septal complex (Fig. 2, middle columns). Subsequently, the same sections were processed with an antibody against $\mathrm{p} 75^{\mathrm{NTR}}$ or ChAT (Fig. 2, right columns), specific markers for cholinergic MSDB neurons (Li et al., 1995). We found that in all mouse strains examined, the distribution of MSDB neurons (Fig. 2, middle columns) that were positive for both $\mathrm{p} 75^{\mathrm{NTR}}$ and ChAT was similar to that of the FG-labeled neurons (Fig. 2, right columns). Hence, our region of interest (Fig. 2 , gray areas in the left columns) for the quantitative analysis of the number of cholinergic neurons can be accurately defined by these three markers. 




Figure 6. Effect of the two p $75^{\mathrm{NTR}}$ mutations at 3 months of age. The persistence of the effect of each $p 75^{\mathrm{NTR}}$ mutation was evaluated at 3 months of age in the congenic B6 strains as described in Figure 5. Although the effects of both mutations are less pronounced with increasing age, the larger increase seen in the $p 75^{\text {exonIV }}$ compared with the p $75^{\text {exonIII }}$ mutation persists.

A striking feature of the different mouse strains, as well as of individuals of the same strain, was the variable location of the MSDB complex along the rostrocaudal axis. This is illustrated in Figure 3, which shows marked differences in the location of the MS region in the forebrain in four representative, different strains. These variations were most pronounced in the Sv129/ BALB/c background of P15 animals and were maintained in part into adulthood. Therefore, traditional anatomic landmarks and stereological coordinates cannot be used to define the exact location of the MS. For example, criteria based on classic landmarks such as "complete crossing of the corpus callosum" (Fig. 3, c.c.) for the first frontal section of a septal series would result in the loss of sections containing parts of the MS, thus yielding artificially reduced cell counts.

\section{Variability of cholinergic MS neurons in the wild-type strains}

During the course of our studies, we also realized that the wild-type littermate groups corresponding to different lines were not identical with regard to the number of neurons in the MS (Albanese et al., 1985; Bentivoglio et al., 1994). Figure 4 shows mean values of ChAT-immunoreactive MS neurons counted with the OF method in the entire septum of 10 mice of each wild-type class. The largest number $(3845 \pm 660)$ corresponds to the wildtype class of the $p 75^{\text {exonIII }}$ line in a mixed background, which also corresponds to the strain with the highest Sv129 content. In contrast, the lowest number $(2402 \pm 188)$ corresponds to the wild-type mice of the $p 75^{\text {exonIV }}$ (B6) line, with a practically $100 \%$ B6 content. Likewise, the congenic $p 75^{\text {exonIII }}(\mathrm{B} 6)$ wild-type mice contain a number of ChAT-immunoreactive MS neurons (2674 \pm 286) that are very similar to those seen for the inbred pure B6 mice $(2892 \pm 484)$. Two strains with intermediate, mixed Sv129/B6 genetic backgrounds that were generated with different clones of Sv129 ES cells yield intermediate numbers of cholinergic neurons (R1, $3471 \pm 574$; J1, $3291 \pm 619)$. From this analysis, then, we conclude that the B6 genetic background contributes significantly to lowering the numbers of ChATimmunoreactive MS neurons (also see next section). In view of these and previous results (Schwegler et al., 1996), it became apparent that a conclusive analysis of cholinergic neurons requires comparison of mutant animals with wild-type littermates of the same line.

\section{Effects of the $p 75^{\text {exonIII }}$ and $p 75^{\text {exonlv }}$ mutations on the number of cholinergic MS neurons at P15}

Ten wild-type and 10 homozygous mutant mice were analyzed at P15 for each $p 75^{N T R}$ mutant line in its original background. This age was selected in light of the largest differences observed in $p 75^{\text {exonIII }}$ mutants in a previous report (Van der Zee et al., 1996). We found that the $p 75^{\text {exonIII }}$ mutation in its original SV129/ BALB/c background leads to only a slight, $6.5 \%$ increase in the number of MS cholinergic neurons, whereas the same analysis performed in the mixed-background $p 75^{\text {exonIV }}$ line reveals that mutant septal regions contain $22 \%$ more neurons than their wild-type littermates (Fig. 5).

To further investigate the effect of the $p 75^{\text {exonIII }}$ mutation in the mixed background, we compared the numbers obtained in individual litters (Fig. 3, Sv129/BALB/c, different colors). This analysis revealed that the effect of targeting exon III of the $p 75^{N T R}$ gene is so subtle that opposing conclusions can be drawn if the number of mice analyzed is too small. Although some differences could be observed within individual litters between p $75^{\text {exonIII }}$ mutants (Fig. 3, top left panel) and their wild-type littermates (Fig. 3, top left panel), no difference could be found when all data for $n=10$ mice of each genotype were pooled. This was primarily attributable to high SD in this group (compare with Fig. 5).

To eliminate the possible effects of two different genetic backgrounds, the same analysis was performed in the two corresponding congenic B6 strains. Here, the $p 75^{\text {exonIII }}$ mutant mice exhibited a $13 \%$ increase over their wild-type littermates, exactly two times the increase observed in the mixed genetic background. In contrast, $p 75^{\text {exonIV }}$ mutant mice show an increase of $22 \%$ (mixed background) or $28 \%$ (B6) with respect to their wild-type littermate controls. In agreement with our observation comparing different wild-type strains, the variability among individuals of the same group was always lower in the congenic B6 strains than in mixed backgrounds (Fig. 5).

Statistical analysis (three-way ANOVA) of these data led to the following conclusions at P15: (1) the genetic background of the mouse (regardless of mutant or wild type) exerts a significant effect $(p<0.0001)$ on the number of cholinergic MS neurons, (2) both mutations in the $p 75^{N T R}$ locus (regardless of which exon was targeted) significantly $(p<0.0001)$ increase this number, and finally, (3) the type of mutation $\left(p 75^{\text {exonIII }} / p 75^{\text {exonIV }}\right)$ significantly $(p<0.05)$ influences this increase.

\section{Late effects of both $p 75^{N T R}$ mutations on the number of MS cholinergic neurons}

We were then interested to see whether the increase in the number of cholinergic neurons determined at P15 would persist with age. Because the largest differences at P15 had been observed in the B6 background, we quantified the effects at 3 months of age in B6 mice. Between P15 and 3 months, there is a slight increase in the number of ChAT-immunoreactive MS neurons in 
Table 1. Genetic background of three representative mice $\left[\mathrm{B} 6, p 75^{\text {exonIII }}(\mathrm{B} 6)\right.$, and $p 75^{\text {exonIV }}$ (B6)], as assessed by microsatellite analysis

\begin{tabular}{|c|c|c|c|c|c|c|c|c|c|}
\hline \multirow[b]{2}{*}{ Marker } & \multicolumn{3}{|l|}{$\underline{\mathrm{C} 57 \mathrm{~B} 16}$} & \multicolumn{3}{|c|}{$p 75^{\text {exonIII }}(\mathrm{BL} 6)$} & \multicolumn{3}{|c|}{$p 75^{\text {exonIV }}(\mathrm{BL} 6)$} \\
\hline & Peak 1 & Peak 2 & Genotype & Peak 1 & Peak 2 & Genotype & Peak 1 & Peak 2 & Genotype \\
\hline D1Mit 15 & 160,08 & 160,08 & C57/C57 & 160,15 & 160,15 & C57/C57 & 160,15 & 160,15 & C57/C57 \\
\hline D1Mit 17 & 167,15 & 167,15 & C57/C57 & 167,07 & 167,07 & C57/C57 & 167,13 & 167,13 & C57/C57 \\
\hline D2Mit 200 & 136,84 & 136,84 & C57/C57 & 136,92 & 136,92 & C57/C57 & 136,93 & 136,93 & C57/C57 \\
\hline D2Mit 5 & 139,91 & 139,91 & C57/C57 & 139,92 & 139,92 & C57/C57 & 139,91 & 139,91 & C57/C57 \\
\hline D3Mit 193 & 149,92 & 149,92 & C57/C57 & 149,92 & 149,92 & C57/C57 & 149,92 & 149,92 & C57/C57 \\
\hline D4Mit 238 & 109,52 & 139,91 & C57/C57 & 139,92 & 139,92 & C57/C57 & 109,79 & 139,91 & C57/C57 \\
\hline D4Mit 42 & 101,71 & 101,71 & C57/C57 & 101,7 & 101,7 & C57/C57 & 101,62 & 101,62 & C57/C57 \\
\hline D5Mit 25 & 233,96 & 233,96 & C57/C57 & 233,92 & 233,92 & C57/C57 & 234,05 & 234,05 & C57/C57 \\
\hline D5Mit 113 & 100,34 & 100,34 & C57/C57 & 100,34 & 100,34 & C57/C57 & 100,34 & 100,34 & C57/C57 \\
\hline D5Mit 152 & 134,35 & 145,08 & $? / \mathrm{C} 57$ & 134,45 & 149,41 & ?/C57 & 128,43 & 134,43 & ?/C57 \\
\hline D5Mit 374 & 116,01 & 116,01 & C57/C57 & 115,92 & 115,92 & C57/C57 & 116,72 & 116,72 & C57/C57 \\
\hline D6Mit 33 & 139,72 & 139,72 & C57/C57 & 139,73 & 139,73 & C57/C57 & 139,64 & 139,64 & C57/C57 \\
\hline D6Mit 138 & 108,87 & 108,87 & C57/C57 & 108,89 & 108,89 & C57/C57 & 108,89 & 108,89 & C57/C57 \\
\hline D6Mit 199 & 141,53 & 150,39 & C57/C57 & 141,6 & 150,39 & C57/C57 & 141,53 & 150,39 & C57/C57 \\
\hline D6Mit 55 & 110,04 & 110,04 & C57/C57 & 109,98 & 109,98 & C57/C57 & 109,99 & 109,99 & C57/C57 \\
\hline D7Mit 129 & 141,53 & 150,39 & $\mathrm{C} 57 / \mathrm{C} 57$ & 141,6 & 150,39 & $\mathrm{C} 57 / \mathrm{C} 57$ & 141,53 & 150,39 & $\mathrm{C} 57 / \mathrm{C} 57$ \\
\hline D7Mit 152 & 126,8 & 126,8 & C57/C57 & 126,83 & 126,83 & C57/C57 & 126,77 & 126,77 & C57/C57 \\
\hline D7Mit 189 & 132,61 & 132,61 & C57/C57 & 132,76 & 132,76 & C57/C57 & 132,62 & 132,62 & C57/C57 \\
\hline D7Mit 284 & 134,61 & 134,61 & C57/C57 & 134,6 & 134,6 & C57/C57 & 134,65 & 134,65 & C57/C57 \\
\hline D7Mit 52 & 166,3 & 166,3 & C57/C57 & 166,39 & 166,39 & C57/C57 & 166,31 & 166,31 & C57/C57 \\
\hline D8Mit 155 & 149,4 & 149,4 & C57/C57 & 149,41 & 149,41 & C57/C57 & 149,4 & 149,4 & C57/C57 \\
\hline D8Mit 166 & 118,84 & 118,84 & C57/C57 & 118,73 & 118,73 & C57/C57 & 118,73 & 118,73 & $\mathrm{C} 57 / \mathrm{C} 57$ \\
\hline D8Mit 93 & 168,55 & 168,55 & C57/C57 & 168,62 & 168,62 & C57/C57 & 168,57 & 168,57 & C57/C57 \\
\hline D9Mit 286 & 109,77 & 109,77 & C57/C57 & 109,75 & 109,75 & C57/C57 & 109,77 & 109,77 & C57/C57 \\
\hline D9Mit 90 & 132,53 & 132,53 & C57 2/C57 2 & 132,51 & 132,51 & C57 2/C57 2 & 132,54 & 132,54 & C57 2/C57 2 \\
\hline D10Mit 86 & 156,1 & 156,1 & C57/C57 & 156,16 & 156,16 & C57/C57 & 156,12 & 156,12 & C57/C57 \\
\hline D10Mit 152 & 141,53 & 150,39 & C57/C57 & 141,6 & 150,39 & C57/C57 & 141,53 & 150,39 & C57/C57 \\
\hline D10Mit 180 & 132,61 & 132,61 & C57/C57 & 132,76 & 132,76 & C57/C57 & 132,62 & 132,62 & C57/C57 \\
\hline D10Mit 205 & 118,93 & 118,93 & C57/C57 & 118,88 & 134,51 & C57/129 Sv* & 118,96 & 118,96 & C57/C57 \\
\hline D10Mit 31 & 152,71 & 152,71 & C57/C57 & 152,79 & 152,79 & C57/C57 & 152,77 & 152,77 & C57/C57 \\
\hline D11Mit 270 & 123,2 & 123,2 & C57/C57 & 111,5 & 123,25 & $129 \mathrm{~Sv} / \mathrm{C} 57^{*}$ & 123,15 & 123,15 & C57/C57 \\
\hline D11Mit 320 & 167,15 & 260,37 & C57/C57 & 167,07 & 260,27 & C57/C57 & 167,13 & 260,4 & C57/C57 \\
\hline D11Mit 71 & 215,3 & 215,3 & C57/C57 & 215,39 & 215,39 & C57/C57 & 215,29 & 215,29 & C57/C57 \\
\hline D12Mit 60 & 145,08 & 149,4 & $\mathrm{C} 57 / \mathrm{C} 57$ & 149,41 & 149,41 & $\mathrm{C} 57 / \mathrm{C} 57$ & 149,4 & 149,4 & $\mathrm{C} 57 / \mathrm{C} 57$ \\
\hline D12Mit 99 & & & & 151,57 & 151,57 & C57/C57 & 151,49 & 151,49 & C57/C57 \\
\hline D13Mit 151 & 130,87 & 130,87 & C57/C57 & 130,95 & 130,95 & C57/C57 & 130,95 & 196,86 & C57/C57 \\
\hline D13Mit 236 & 95,95 & 95,95 & C57/C57 & 96,03 & 96,03 & C57/C57 & 85,92 & 95,95 & $129 \mathrm{~Sv} / \mathrm{C} 57^{*}$ \\
\hline D13Mit 97 & 152,27 & 152,27 & C57/C57 & 152,27 & 152,27 & C57/C57 & 152,2 & 152,2 & C57/C57 \\
\hline D14Mit 192 & 263,07 & 263,07 & C57/C57 & 262,98 & 262,98 & C57/C57 & 262,96 & 262,96 & C57/C57 \\
\hline D14Mit 99 & 123,73 & 123,73 & C57/C57 & 123,73 & 123,73 & C57/C57 & 123,76 & 123,76 & C57/C57 \\
\hline D15Mit 12 & 151,57 & 151,57 & C57/C57 & 151,57 & 151,57 & C57/C57 & 151,5 & 151,5 & C57/C57 \\
\hline D15Mit 8 & 132,61 & 160,08 & C57/C57 & 132,76 & 160,15 & C57/C57 & 132,62 & 160,15 & C57/C57 \\
\hline D16Mit 152 & 107,38 & 107,38 & C57/C57 & 107,43 & 107,43 & C57/C57 & 107,37 & 107,37 & C57/C57 \\
\hline D16Mit 106 & 152,71 & 152,71 & C57/C57 & 152,79 & 152,79 & C57/C57 & 152,77 & 152,77 & C57/C57 \\
\hline D16Mit 4 & 130,87 & 130,87 & C57/C57 & 130,95 & 130,95 & C57/C57 & 130,95 & 130,95 & C57/C57 \\
\hline D17Mit 129 & 149,4 & 149,4 & C57/C57 & 149,57 & 149,57 & C57/C57 & 149,57 & 149,57 & C57/C57 \\
\hline D17Mit 164 & 132,18 & 132,18 & C57/C57 & 132,17 & 132,17 & C57/C57 & 132,17 & 132,17 & C57/C57 \\
\hline D17Mit 176 & 170,2 & 170,2 & C57/C57 & 170,17 & 170,17 & C57/C57 & 170,28 & 170,28 & C57/C57 \\
\hline D17Mit 93 & 156,23 & 156,23 & C57/C57 & 156,31 & 156,31 & C57/C57 & 156,27 & 156,27 & C57/C57 \\
\hline D18Mit 19 & 153,72 & 153,72 & C57/C57 & 153,65 & 153,65 & C57/C57 & 153,71 & 153,71 & C57/C57 \\
\hline D18Mit 25 & 123,86 & 127,29 & ?/C57 & 123,78 & 127,2 & ?/C57 & 123,78 & 127,22 & ?/C57 \\
\hline D18Mit 23 & 145,08 & 145,08 & C57/C57 & 145,02 & 145,02 & C57/C57 & 145,08 & 145,08 & C57/C57 \\
\hline D18Mit 33 & 140,45 & 140,45 & C57/C57 & 140,46 & 140,46 & C57/C57 & 140,45 & 140,45 & C57/C57 \\
\hline D19Mit 119 & 260,37 & 260,37 & C57/C57 & 260,27 & 260,27 & C57/C57 & 260,4 & 260,4 & C57/C57 \\
\hline D19Mit 78 & 136,01 & 136,01 & C57/C57 & 135,92 & 135,92 & C57/C57 & 135,93 & 135,93 & C57/C57 \\
\hline D19Mit 91 & 108,61 & 108,61 & C57/C57 & 108,63 & 108,63 & C57/C57 & 108,64 & 108,64 & C57/C57 \\
\hline DXMit 222 & 170,2 & 170,2 & C57/C57 & 170,17 & 170,17 & C57/C57 & 170,28 & 170,28 & C57/C57 \\
\hline DXMit 62 & 124,58 & 124,58 & C57/C57 & 124,39 & 124,39 & C57/C57 & 124,46 & 124,46 & C57/C57 \\
\hline DXMit 149 & 276 & 276 & C57/C57 & 276,37 & 276,37 & C57/C57 & 276,18 & 276,18 & C57/C57 \\
\hline
\end{tabular}

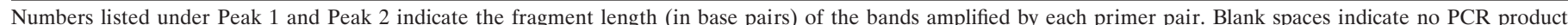

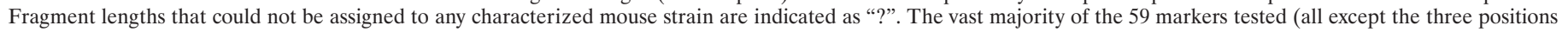
marked *) showed homozygosity for the B6 genotype (C57/C57). 




Figure 7. Differential expression of the s-p $75^{\mathrm{NTR}}$ isoform in different genetic backgrounds. The accumulation of the s-p $75^{\mathrm{NTR}}$ mRNA was analyzed by reverse transcription-PCR in P15 whole brains and in MS from B6 and Sv129 mice. Unlike the FL-p $75^{\text {NTR }}$ mRNA, which accumulates at comparable levels in both strains, in whole brain the s-p $75^{\mathrm{NTR}}$ transcript accumulates at much higher levels in Sv129 than in B6 animals. Shown here are the results from the MS (pooled from 5 mice of each strain), in which s-p $75^{\mathrm{NTR}}$ accumulates at $>10$-fold higher levels in the Sv129 background.

wild-type animals of both mutant lines: from $2674 \pm 286$ to $2808 \pm 327$ for the $p 75^{\text {exonIII }}$ mutation and from $2402 \pm 188$ to $2520 \pm 329$ for the $p 75^{\text {exonIV }}$ mutation (Fig. 6). This is in agreement with counts performed in the inbred C57BL/6 strain at P15 and 3 months of age, when no major changes were observed (data not shown). In contrast, the number of MS cholinergic neurons shows a tendency to decrease in both $p 75^{N T R}$ mutants: from $3026 \pm 328(\mathrm{P} 15)$ to $2963 \pm 271$ (3 months) for the $p 75^{\text {exonIII }}$ mutation and from $3082 \pm 258$ (P15) to $2796 \pm 418$ ( 3 months) for the $p 75^{\text {exonIV }}$ mutation. There are $5.5 \%$ more cholinergic neurons in the $p 75^{\text {exonIII }}$ mutants than in their wild-type littermates, and $11 \%$ more for $p 75^{\text {exonIV }}$ mutants.

Statistical analysis (three-way ANOVA) of these data revealed that (1) the age of the animal significantly $(p<0.0001)$ influences the number of cholinergic neurons and that (2) the difference between wild-type and mutants (regardless of which exon was targeted) is maintained $(p<0.0026)$. The differential effects of the two mutations, however, are now less pronounced $(p<$ $0.0661)$.

\section{The accumulation of the s-p $75^{\mathrm{NTR}}$ transcript is strain dependent}

Because the only difference between $p 75^{\text {exonIII }}$ and $p 75^{\text {exonIV }}$ mutants at the molecular level is the presence of a residual s-p $75^{\text {NTR }}$ transcript in the former mice, we tested whether the strain-dependent phenotype observed in the MS could be correlated with strain-dependent levels of spliced transcript. Reverse transcription-PCR analysis performed with whole-brain RNA shows that at P15, the s-p75 NTR variant is more abundant in Sv129 mice than in their B6 counterparts, whereas the levels of FL-p75 ${ }^{\text {NTR }}$ transcript in whole brain are comparable among mice of both strains. At P15, the MS is one of the brain regions with the highest accumulation levels of s-p $75^{\text {NTR }}$ and one of the regions where its higher expression levels in Sv129 background can be most clearly seen (Fig. 7).

\section{DISCUSSION}

The main result of our study is that mice lacking $\mathrm{p} 75^{\mathrm{NTR}}$ have significantly more cholinergic neurons in the MS. This result is in line with previous reports demonstrating that one of the functions of p $75^{\text {NTR }}$ is to cause cell death during development, as reported previously in the developing retina and the spinal cord as well as in peripheral sympathetic ganglia (Frade et al., 1996; Bamji et al., 1998; Frade and Barde, 1999). Because the role of $\mathrm{p} 75^{\mathrm{NTR}}$ in the development of basal forebrain cholinergic neurons has been analyzed in previous studies that led to contradictory results (see the introductory remarks), the most likely reasons for these discrepancies are discussed below.

\section{Distribution of cholinergic septal neurons in the MS and influence of genetic background}

A major difficulty encountered when comparing previous results is that the region of interest, the MS nucleus, has often been determined on the basis of somewhat subjective criteria. The septal region is composed of several different nuclei, and it is heterogeneous with regard to its neuronal composition and connectivity. Moreover, the cholinergic neurons represent only a small fraction of the neurons in the MSDB complex. Because it was unclear whether in the mouse, as established previously in the rat (Sofroniew et al., 1987, 1990), most cholinergic MS neurons would project to the hippocampal formation, we analyzed their projections in mice of all genotypes using FG back-tracing. A strong overlap between the distribution of back-labeled, large magnocellular neurons and neurons immunoreactive for both p75 ${ }^{\text {NTR }}$ and ChAT could be observed in all mouse strains. Although these experiments also revealed that the anatomy of the MSDB is very similar regardless of the strain investigated, the dimension of the region containing FG back-labeled neurons varied substantially along the rostrocaudal axis. Therefore, traditional anatomic landmarks and stereological coordinates cannot be used to define the exact location of the MS. To define the total area of the region of interest and its reference volume, we avoided the use of "classic" anatomic boundaries of the septal complex, such as the corpus callosum, the ventral surface of the brain, and "straight lines" drawn from the ventral tips of the lateral ventricles to the ventral surface of the brain (Peterson et al., 1999). Whereas the boundaries of the MSDB region to lateral and dorsal nuclei of the septal complex can be easily marked using version 3 of the Stereo Investigator Software, any delineation of the MS against the DB tends to be arbitrary (Jakab and Leranth, 1995), because neurons of the MSDB form a continuum and are the rostral part of the large basal forebrain region. Therefore, we delineated the MS against the DB by a horizontal line connecting the edges of the anterior commissure, as shown in Figure 2.

Cholinergic neurons were also regularly found in the neighboring lateral septal nucleus. Although rather small, they sometimes form dense clusters, and nearly all of them are negative when stained with $\mathrm{p} 75^{\mathrm{NTR}}$ antibodies. In contrast, adjacent telencephalic nuclei located more ventrocaudally (Fig. 2, position 16) contain ChAT-immunoreactive neurons that are also $\mathrm{p} 75^{\mathrm{NTR}}$. immunopositive; however, they do not belong to the MS nucleus. All of these neurons may have been included, at least partially, in some of the previous studies. Using our criteria, and despite the rostrocaudal variability seen between different animals, we found that the MS region of P15 mice consistently yielded a total of 22-24 consecutive frontal vibratome sections. A slightly higher number of frontal sections was obtained from 3-month-old ani- 
mals, which was in line with a slight increase of the reference volume observed with increasing age.

A major outcome of our investigations in wild-type animals is that the number of cholinergic neurons in the MS strongly depends on the genetic background. We found that at P15, pure B6 animals have $\sim 33 \%$ fewer cholinergic neurons than Sv129 animals (Fig. 4). This background-dependent effect is in fact larger than the largest difference we observed with the $p 75^{N T R}$ mutant animals (see below).

\section{The $p 75^{\text {exon/V }}$ mutation leads to larger increases of cholinergic neurons than the $p 75^{\text {exonllI }}$ mutation}

As we observed previously in the peripheral nervous system (von Schack et al., 2001), the $p 75^{\text {exonIV }}$ mutation has a significantly higher impact on the number of MS cholinergic neurons than the $p 75^{\text {exonIII }}$ mutation. The higher degree of variability among individual animals carrying the $p 75^{\text {exonIII }}$ mutation, especially in their original genetic background, also parallels the variability found in counts of sensory neurons (von Schack et al., 2001). Both the variability and the relatively small effects may have obscured the interpretation of previous studies, all performed with the $p 75^{\text {exonIII }}$ mutation. In contrast, the $p 75^{\text {exonIV }}$ mutation displays a robust phenotype that is largely independent of the genetic background. Because the major difference is that the $p 75^{\text {exonIII }}$ mutation allows residual expression of the s-p $75^{\mathrm{NTR}}$ isoform, it is possible that $s-p 75^{\text {NTR }}$ partially compensates for the lack of FL-p $75^{\text {NTR }}$. Indeed, the cytoplasmic domain of p $75^{\text {NTR }}$, although expressed at much reduced levels compared with wild-type animals, is still intact and most likely still binds the various cytoplasmic interactors of $\mathrm{p} 75^{\mathrm{NTR}}$ and interacts with Trk receptors (Bibel et al., 1999; von Schack et al., 2001; for review, see Bibel and Barde, 2000). It is therefore of interest that s-p $75^{\text {NTR }}$ is present at substantially higher levels in the Sv129 background compared with the B6 brain. This may contribute to explaining why the increase in MS cholinergic neurons is smaller in the Sv129 background than in the congenic B6 strain. This reasoning would imply that the effects of the $p 75^{\text {exonIV }}$ mutation, which completely abolishes expression of s-p $75^{\mathrm{NTR}}$, should not depend on genetic background. In line with this, the mixed B6/Sv129 strain does indeed show an effect of comparable magnitude to that seen in the congenic B6 strain.

Because previous experiments indicate that $\mathrm{p} 75^{\mathrm{NTR}}$ can cause cell death (Casaccia-Bonnefil et al., 1996; Frade et al., 1996; Bamji et al., 1998; Yoon et al., 1998; Frade and Barde, 1999), it appears likely that the explanation for the increased number of cholinergic neurons is a reduction in cell death. Also, previous work has demonstrated that overexpression of the intracellular domain of $\mathrm{p} 75^{\mathrm{NTR}}$ leads to massive cell death throughout the CNS (Majdan et al., 1997). This is strong evidence that the cytoplasmic domain of $\mathrm{p} 75^{\mathrm{NTR}}$ has a substantial death potential for CNS neurons and would be consistent with our interpretation that the complete elimination of the receptor, including its cytoplasmic domain, decreases cell death. How p75 NTR causes cell death is still not understood in detail, but at least one of the interactors of $\mathrm{p} 75^{\mathrm{NTR}}$, the neurotrophin receptor interacting factor protein, has been shown to be involved in cell death in the developing CNS (Casademunt et al., 1999). It is also possible that the complete lack of p $75^{\mathrm{NTR}}$ increases the efficiency of signaling through TrkA. TrkA is coexpressed with $\mathrm{p} 75^{\mathrm{NTR}}$ in most cholinergic neurons, and TrkA-/- mice have reduced numbers of MS cholinergic neurons (Fagan et al., 1997). In this context, it is worth noting that both full-length and s-p $75^{\text {NTR }}$ and Trk recep- tors interact (Bibel et al., 1999; von Schack et al., 2001), and there is evidence that $\mathrm{p} 75^{\mathrm{NTR}}$ decreases Trk signaling (for review, see Kaplan and Miller, 1997; Bibel and Barde, 2000).

In conclusion, our results show that the complete absence of p $75^{\text {NTR }}$ leads to a persistent increase in the number of cholinergic neurons in the MS of the mouse. Given the significance of cholinergic forebrain neurons in learning and memory processes and their degeneration in Alzheimer's disease, our results indicate that a cell-surface receptor expressed at comparatively high levels by these neurons may be an interesting target with regard to the modulation of the survival of these neurons.

\section{REFERENCES}

Albanese A, Gozzo S, Iacopino C, Altavista MC (1985) Straindependent variations in the number of forebrain cholinergic neurons. Brain Res 334:380-384.

Bamji SX, Majdan M, Pozniak CD, Belliveau DJ, Aloyz R, Kohn J, Causing CG, Miller FD (1998) The p75 neurotrophin receptor mediates neuronal apoptosis and is essential for naturally occurring sympathetic neuron death. J Cell Biol 140:911-923.

Bartus RT, Dean RL, Beer B, Lippa AS (1982) The cholinergic hypothesis of geriatric memory dysfunction. Science 217:408-414

Bentivoglio AR, Altavista MC, Granata R, Albanese A (1994) Genetically determined cholinergic deficiency in the forebrain of $\mathrm{C} 57 \mathrm{BL} / 6$ mice. Brain Res 637:181-189.

Bibel M, Barde Y-A (2000) Neurotrophins: key regulators of cell fate and cell shape in the vertebrate nervous system. Genes Dev 14:2919-2937.

Bibel M, Hoppe E, Barde Y-A (1999) Biochemical and functional interactions between the neurotrophin receptors trk and $\mathrm{p} 75^{\mathrm{NTR}}$. EMBO J $18: 616-622$.

Casaccia-Bonnefil P, Carter BD, Dobrowsky RT, Chao MV (1996) Death of oligodendrocytes mediated by the interaction of nerve growth factor with its receptor p75. Nature 383:716-719.

Casademunt E, Carter BD, Benzel I, Frade JM, Dechant G, Barde Y-A (1999) The zinc finger protein NRIF interacts with the neurotrophin receptor p75(NTR) and participates in programmed cell death. EMBO J 18:6050-6061.

Coyle JT, Price DL, DeLong MR (1983) Alzheimer's disease: a disorder of cortical cholinergic innervation. Science 219:1184-1190.

Fagan AM, Garber M, Barbacid M, Silos-Santiago I, Holtzman DM (1997) A role for TrkA during maturation of striatal and basal forebrain cholinergic neurons in vivo. J Neurosci 17:7644-7654.

Fischer W, Wictorin K, Björklund A, Williams LR, Varon S, Gage FH (1987) Amelioration of cholinergic neuron atrophy and spatial memory impairment in aged rats by nerve growth factor. Nature 329:65-68.

Frade JM, Barde Y-A (1999) Genetic evidence for cell death mediated by nerve growth factor and the neurotrophin receptor $\mathrm{p} 75$ in the developing mouse retina and spinal cord. Development 126:683-690.

Frade JM, Rodriguez-Tébar A, Barde Y-A (1996) Induction of cell death by endogenous nerve growth factor through its p75 receptor. Nature 383:166-168.

Franklin KB, Paxinos G (1997) The mouse brain. San Diego: Academic.

Green MC, Witham BA (1991) Handbook of genetically standardized JAX mice. Bar Harbor, ME: The Jackson Laboratory.

Greferath U, Bennie A, Kourakis A, Bartlett PF, Murphy M, Barrett GL (2000) Enlarged cholinergic forebrain neurons and improved spatial learning in p75 knockout mice. Eur J Neurosci 12:885-893.

Hagg T (1999) Neuronal cell death: retraction. Science 285:340.

Hagg T, Louis J-C, Longo FM, Varon S (1994) Neurotrophic factors, growth factors, and central nervous system trauma. In: The neurobiology of the central nervous system trauma (Salzman SK, Faden IA, eds), pp 245-265. Oxford: Oxford UP.

Hagg T, Van der Zee CEEM, Ross GM, Riopelle RJ (1997) Basal forebrain neuronal loss in mice lacking neurotrophin receptor p75. Science 277:838-839.

Hefti F (1986) Nerve growth factor promotes survival of septal cholinergic neurons after fimbrial transections. J Neurosci 6:2155-2162.

Jakab RL, Leranth C (1995) Septum. In: The rat nervous system (Paxinos G, ed), pp 405-442. Oxford: Academic.

Kaplan DR, Miller FD (1997) Signal transduction by the neurotrophin receptors. Curr Opin Cell Biol 9:213-221.

Lee KF, Li E, Huber LJ, Landis SC, Sharpe AH, Chao MV, Jaenisch R (1992) Targeted mutation of the gene encoding the low affinity NGF receptor $\mathrm{p} 75$ leads to deficits in the peripheral sensory nervous system. Cell 69:737-749.

Li Y, Holtzman DM, Kromer LF, Kaplan DR, Chua-Couzens J, Clary DO, Knusel B, Mobley WC (1995) Regulation of TrkA and ChAT expression in developing rat basal forebrain: evidence that both exog- 
enous and endogenous NGF regulate differentiation of cholinergic neurons. J Neurosci 15:2888-2905.

Lucidi-Phillipi CA, Clary DO, Reichardt LF, Gage FH (1996) TrkA activation is sufficient to rescue axotomized cholinergic neurons. Neuron 16:653-663.

Majdan M, Lachance C, Gloster A, Aloyz R, Zeindler C, Bamji S, Bhakar A, Belliveau D, Fawcett J, Miller FD, Barker PA (1997) Transgenic mice expressing the intracellular domain of the p75 neurotrophin receptor undergo neuronal apoptosis. J Neurosci 17:6988-6998.

Naumann T, Linke R, Frotscher M (1992) Fine structure of rat septohippocampal neurons. I. Identification of septohippocampal projection neurons by retrograde tracing combined with electron microscopic immunocytochemistry and intracellular staining. J Comp Neurol 325:207-218

Naumann T, Kermer P, Frotscher M (1994) Fine structure of rat septohippocampal neurons. III. Recovery of choline acetyltransferase immunoreactivity after fimbria-fornix transection. J Comp Neurol 350:161-170.

Naumann T, Straube A, Frotscher M (1997) Recovery of ChAT immunoreactivity in axotomized rat cholinergic septal neurons despite reduced NGF receptor expression. Eur J Neurosci 9:1340-1349.

Perry E, Walker M, Grace J, Perry R (1999) Acetylcholine in mind: a neurotransmitter correlate of consciousness? Trends Neurosci 6:273-280.

Peterson DA, Leppert JT, Lee KF, Gage FH (1997) Basal forebrain neuronal loss in mice lacking neurotrophin receptor p75. Science 277:837-839.

Peterson DA, Dickinson-Anson HA, Leppert JT, Lee KF, Gage FH (1999) Central neuronal loss and behavioral impairment in mice lacking neurotrophin receptor p75. J Comp Neurol 404:1-20.

Schwegler H, Boldyreva M, Pyrlik-Gohlmann M, Linke R, Wu J, Zilles K (1996) Genetic variation in the morphology of the septo-hippocampal cholinergic and GABAergic system in mice. I. Cholinergic and GABAergic markers. Hippocampus 6:136-148.

Simpson EM, Linder CC, Sargent EE, Davisson MT, Mobraaten LE,
Sharp JJ (1997) Genetic variation among 129 substrains and its importance for targeted mutagenesis in mice. Nat Genet 16:19-27.

Sofroniew MV, Pearson RC, Powell TP (1987) The cholinergic nuclei of the basal forebrain of the rat: normal structure, development and experimentally induced degeneration. Brain Res 411:310-331.

Sofroniew MV, Galletly NP, Isacson O, Svendsen CN (1990) Survival of adult basal forebrain cholinergic neurons after loss of target neurons. Science 247:338-342.

Van der Zee CEEM, Ross GM, Riopelle RJ, Hagg T (1996) Survival of cholinergic forebrain neurons in developing p75NGFR-deficient mice. Science 274:1729-1732.

Vantini G, Schiavo N, Di Martino A, Polato P, Triban C, Callegaro L, Toffano G, Leon A (1989) Evidence for a physiological role of nerve growth factor in the central nervous system of neonatal rats. Neuron 3:267-273.

von Schack D, Casademunt E, Schweigreiter R, Meyer M, Bibel M, Dechant G (2001) Complete ablation of the neurotrophin receptor p $75^{\text {NTR }}$ causes defects both in the nervous and the vascular systems. Nat Neurosci 4:1-2.

Wahlsten D (1982) Deficiency of corpus callosum varies with strain and supplier of the mice. Brain Res 239:329-347.

Wahlsten D, Bulman-Fleming B (1994) Retarded growth of the medial septum: a major gene effect in acallosal mice. Dev Brain Res 77:203-214.

Ward NL, Hagg T (1999) p75(NGFR) and cholinergic neurons in the developing forebrain: a re-examination. Dev Brain Res 118:79-91.

West MJ, Slomianka L, Gundersen HJ (1991) Unbiased stereological estimation of the total number of neurons in the subdivisions of the rat hippocampus using the optical fractionator. Anat Rec 231:482-497.

Yeo TT, Chua-Couzens J, Butcher LL, Bredesen DE, Cooper JD, Valletta JS, Mobley WC, Longo FM (1997) Absence of p75NTR causes increased basal forebrain cholinergic neuron size, choline acetyltransferase activity, and target innervation. J Neurosci 17:7594-7605.

Yoon SO, Casaccia-Bonnefil P, Carter B, Chao MV (1998) Competitive signaling between TrkA and p75 nerve growth factor receptors determines cell survival. J Neurosci 18:3273-3281. 